\title{
Inhalt des Heftes 6
}

Wenzel, J., Kirsche, W., Kunz, G., Neumann, H., Wenzel, M. und Winkelmann, E., Licht- und elektronenmikroskopische Untersuchungen über die Dendritenspines an Pyramiden-Neuronen des Hippocampus (CA 1) bei der Ratte . . . . . . . . . . . . . . . . . . . . . . . . . . . . . . . . . . . 387

Schnitzlern, H. N., Hamel, E. G., Carey, J. H., Brown, J. W., Hoffman, H. H., Haucette, J. R., and Showirs, M. J. C., The interrelations of the striatum with subcortical areas through the lateral forebrain bundle 409

YAMAdORI, T., An experimental anatomical study of the fasciculus mamillothalamicus in rats . . . . . . . . . . 457

Poliakov, G. I., Some morphological aspects of the integrating activity of the cerebral cortex . . . . . . . . . 469

Voth, D., Müller, D. und Possner, R., Vergleichende Untersuchungen über den Sauerstoffverbrauch, den Elektrolytstoffwechsel und die Ultramorphologie des isolierten Plexus chorioideus des Kaninchens . . 489

Burri, P. H. und Schneider, H. R., Anwendung cytoarchitektonischer Kriterien für die Neuronentypisierung in der Elektronenmikroskopie . . . . . . . . . . . . . . . . . . . . . . . 509

Im JOURNAL FUUR HIRNFORSCHUNG werden Arbeiten aus dem Gesamtgebiet der normalen Morphologie (Anatomie, Histologie, Cytologie, Elektronenmikroskopie, Histochemie) und der Entwicklungsgeschichte des Nervensystems unter Einschluß experimentell-anatomischer Arbeiten veröffentlicht. Neuropathologische Arbeiten werden nur angenommen, wenn sie Beiträge zur normalen Struktur, den Strukturwandlungen oder deren funktionellen Bedeutungen enthalten. Zum Publikationsgebiet des JOURNAL FUER HIRNFORSCHUNG gehören auch Arbeiten, die sich mit der Zuordnung experimenteller Reiz- und Ausfallerscheinungen bzw. klinischen Symptomen zu bestimmten Strukturen des Gehirns (,Lokalisationslehre“) befassen. Als spezielles Publikationsgebiet ist die vergleichende Neurobiologie vorgeschen.

The JOURNAL FÚR HIRNFORSCHUNG will,publish studies on normal morphology (anatomy, histology, cytology, electron microscopy, histochemistry), on the development of the nervous system, as well experimental anatomical studies. Neuropathological studies will only be published if they contribute to the knowledge of normal structures, structural changes or their functional significance. Papers dealing with the cerebral localization of experimental excitation and deficit phenomena or clinical symptoms (localization theory) will also be published by the JOURNAL FUER HIRNFORSCHUNG. A special part of the publication is reserved for comparative neurobiology.

Le JOURNAL FU'R HIRNFORSCHUNG publierą des études sur la morphologie normale (anatomie, histologie, cytologie, microscopie électronique, histochimie), sur le développement du système nerveux ainsi que des études anatomiques expérimentales. Des études neuropathologiques seront seulement acceptées quand elles contribuent à la connaissance des structures normales, des changements structurels ou de leur signification fonctionelle. Des études sur la localisation cérebrale de phénoménes expérimentaux ou cliniques d'excitation ou de déficit (dọctrine des localisations) seront également publiées par le JOURNAL FUER HIRNFORSCHUNG. Une partie spéciale sera réservée à la neurobiologie comparee.

\footnotetext{
Verantwortlích für den Inhalt Prof. Dr. I. Anthony, Paris; Prof. Dr. A. Hopf, 4 Düsscldorf; Prof. Dr. W. Kirsche, 104 Berlin; Prof. Dr. J. Szentágothai, Budapest. Verlag: Akademie-Verlag GmbH, 108 Berlin, Leipziger Straße 3-4.

Fcrnruf: 200441, Telex-Nr. 011 2020; Postscheckkonto: Berlin 35021. Bestellnummer dieses Heftes: 1018/13/6.

Das ,.Journal für Hirnforschung “ erscheint sechsmal jährlich. 6 Hefte bilden einen Band.

Preis je Einzelheft 25,-M. Der Preis für einen Band beträgt 150,- M.

Satz und Druck: VEB Druckhauis „Maxim Gorki“", 74 Altenburg.

Veröffentlicht unter der Lizenznummer 1326 des Presseamtes beim Vorsitzenden des Ministerrates der Deutschen Demokratischen Republik.

Printed in the German Democratic Republic
} 